\title{
Plasmonic properties of gold nanospheres coupled to reduced graphene oxide for biosensing applications*
}

\author{
Alessandro Fantoni, Vladan Stojkovic, Miguel Fernandes, Manuela Vieira, Elisabete C.B.A. Alegria, \\ Ana P.C. Ribeiro
}

\begin{abstract}
Graphene-based materials have been extensively explored in recent years as valuable candidates as the key material for novel structures in the field, among many other applications, of sensing devices. Reduced Graphene Oxide (rGO) is a type of chemically derived graphene, with equivalent optical properties but easier to be synthetized. This work reports a study about the applicability of rGO as a support for gold nanoparticles (AuNPs). The resulting AuNPs-rGo composites are studied in terms of spectral light transmission and plasmonic resonance as a possible sensing element for a photonic protein sensor device.
\end{abstract}

\section{INTRODUCTION}

Because of its interesting optical, thermal and mechanical properties, graphene has found during the last years its path in a large variety of biosensing applications [1]. Recently, reduced Graphene Oxide (rGO) has been proposed and studied as a nanocomposite in conjunction with gold nanoparticles [2] and also proposed as a biosensing element for aflatoxins [3]. rGO is studied in this work in conjunction with the local surface plasmonic resonance (LSPR) produced by gold nanospheres (AuNPs) and we present results about light transmission and absorption of rGO decorated with AuNPs, evaluating the possibility of using this material as the sensing element for a protein sensor device.

The proposed device structure is depicted in Figure 1. It is a plasmonic structure based on the LSPR interaction of $\mathrm{Au}$ nanoparticles, embedded into a matrix of rGO. After proper functionalization with selective antibodies, and for a tuned wavelength, light transmission is controlled by slight changes of the refractive index induced by the biomarker concentration. The use of a functionalized surface based on rGO, allows an improvement of the biocompatibility and a cost reduction. Integration with an a-Si:H photodetector allows the quantification of biomarker fingerprint on the sensing surface. The optoelectronic readout is performed by a pin a-Si:H photodiode. The goal of the work hereby presented is the study of the plasmonic properties of the AuNPs-rGO composites for different light wavelength and nanosphere dimension. The focus of this study is on the LSPR absorption profile, measured by UV-Vis spectroscopic analysis. A theoretical analysis is also presented, based on the Mie theory, about the LSPR of

*Research supported by EU funds through the FEDER European Regional Development Fund and by Portuguese national funds by FCT -Fundação para a Ciência e a Tecnologia with projects PTDC/NAN-OPT/31311/2017, SFRH/BPD/102217/2014 and by IPL IDI\&CA/2018/aSiPhoto.

A. Fantoni is with the CTS-UNINOVA institute, Caparica, Portugal and with ADEETC-ISEL-Instituto Politécnico de Lisboa, Portugal (e-mail: afantoni@deetc.isel.ipl.pt. V. Stojkovic is with ADEETC-ISEL-Instituto
AuNPs with different dimension, diluted in water and in composition with rGO.

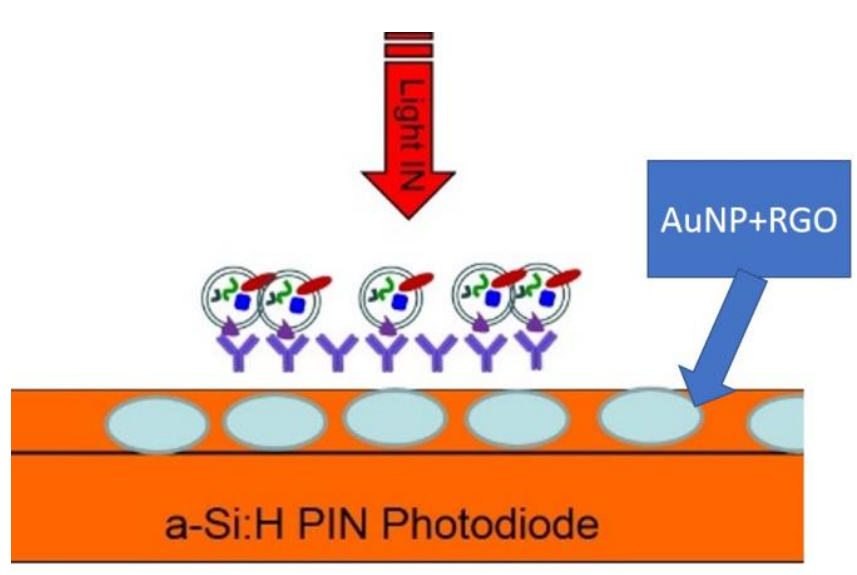

Figure 1. Schematics of the LSPR sensor based on rGO+AuNPS light trasmission properties

\section{Simulation: Mie ANAlysis}

\section{A. Theory}

Even if the plasmonic phenomena were known since the ancient ages, a first theoretical explanation, based on general electromagnetic approach, has been presented only in the beginning of the XX century by Gustave Mie [4]. This theory is still a solid basis for a satisfactory understanding of these phenomena and it is extensively reported [5]. According to the Mie theory, the interaction between light and the nanospheres can be described in terms of the extinction efficiency $\left(Q_{e x t}\right)$, defined as the ratio of the cross-section for light extinction process, to the geometrical section area of the spherical particle. Light extinction is defined as the sum of the two processes describing light absorption and scattering, introducing the analogue quantities of absorption $\left(Q_{a b s}\right)$ and scattering $\left(Q_{s c a}\right)$ efficiency. The Mie theory can be directly applied only to the special case of a spherical particle, admitting an extension to the case of a core-shell sphere. It describes in a very complete way the extinction of a plane

Politécnico de Lisboa, Portugal. M. Fernandes is with the CTS-UNINOVA institute, Caparica, Portugal and with ADEETC-ISEL-Instituto Politécnico de Lisboa, Portugal. M. Vieira is with the CTS-UNINOVA institute, Caparica, Portugal, with DEE-FCT-UNL Caparica, Portugal and with ADEETC-ISEL-Instituto Politécnico de Lisboa, Portugal, E.C.B.A. Alegria is with ADEQ-ISEL-Instituto Politécnico de Lisboa, Portugal and with CQE, Lisbon, Portugal. A.P.C Ribeiro is with CQE, Lisbon, Portugal. 
wave without any restriction to the particle size. The Mie analysis requires the values of the complex refractive index $(n, k)$ for the metal and the surrounding medium. Optical properties for metals and dielectrics, necessary for our simulations, have been taken by literature $[6,7,8,9]$.
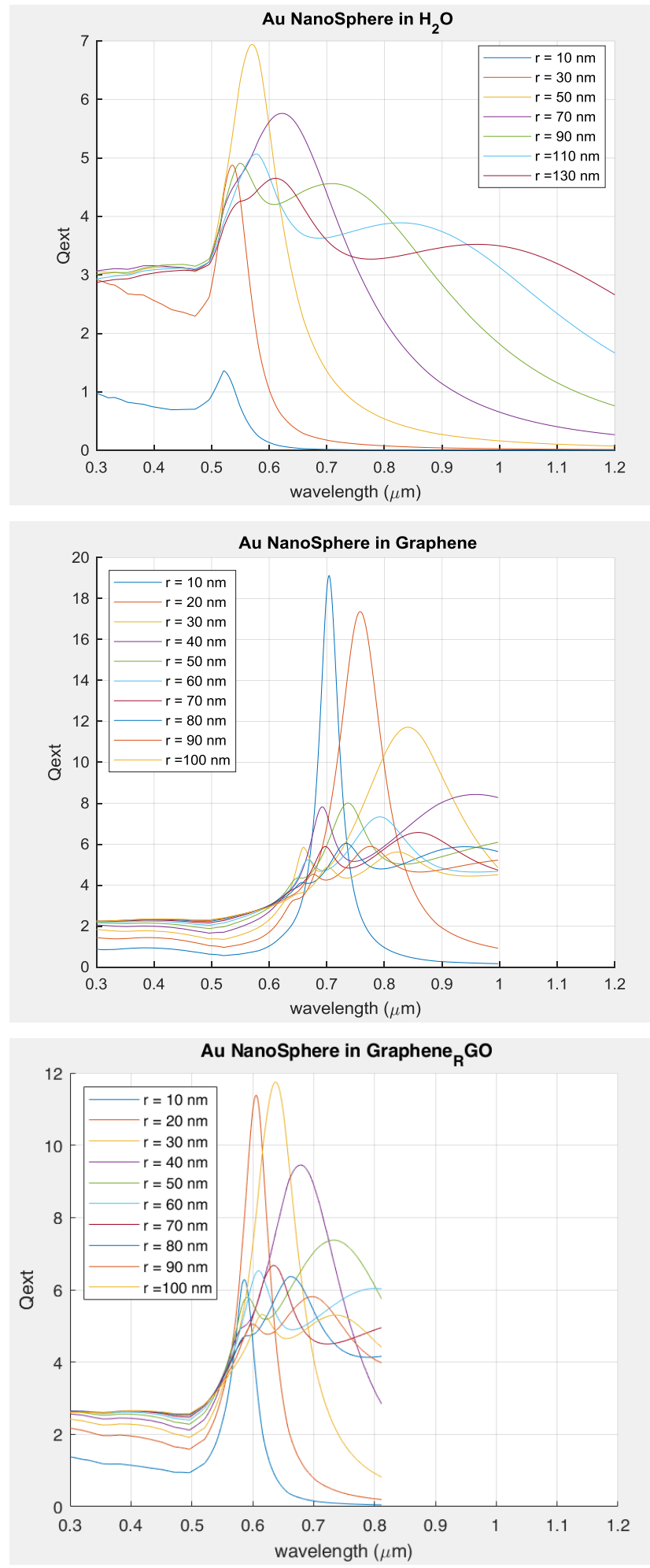

Figure 2. Extinction efficiency, calculated by Mie analysis, for gold nanospheres with radius between 10 and $130 \mathrm{~nm}$ embedded in graphene, $\mathrm{rGO}$ and diluted in water.

\section{B. Simulations}

A Direct Mie analysis has been used to simulate the LSPR of gold nanospheres embedded in Graphene, rGO and, for comparative purpose, in a colloidal solution [10]. As expected, our results show that the wavelength for the LSPR peak depends strongly on the nanoparticle radius. It is also possible to detect an increasing multipolar response and a peak broadening for large sphere radius. On the other hand, a sharp and localized peak can be observed when the NPs radius is small. When the size of the NPs is as small as $20 \mathrm{~nm}$, absorption is the dominant phenomena and the most part of light extinction for these cases is due to the enhance absorption caused by the LSPR. The simulated light extinction for $\mathrm{Au}$ nanospheres with a radius in the range 10-130 nm embedded in Graphene, rGO and distilled water is depicted in Figure 2. The resonance peaks wavelength and width vary greatly with the surrounding medium. In rGO the peak is located in the red part of the spectrum, in the 600-700 range, depending on the NP size. This result is of greatly importance for our projected applications, as the spectral sensitivity of a-Si:H is still good in this range, becoming much lower when entering in the infrared region (wavelengths above $700 \mathrm{~nm}$ ). The extinction efficiency of a $40 \mathrm{~nm}$ nanosphere covered by a thin layer of rGO with increasing thickness is presented in Figure 3. The rGO cladding layer produces the effect of red-shifting the LSPR peak, proportionally to the cladding thickness.

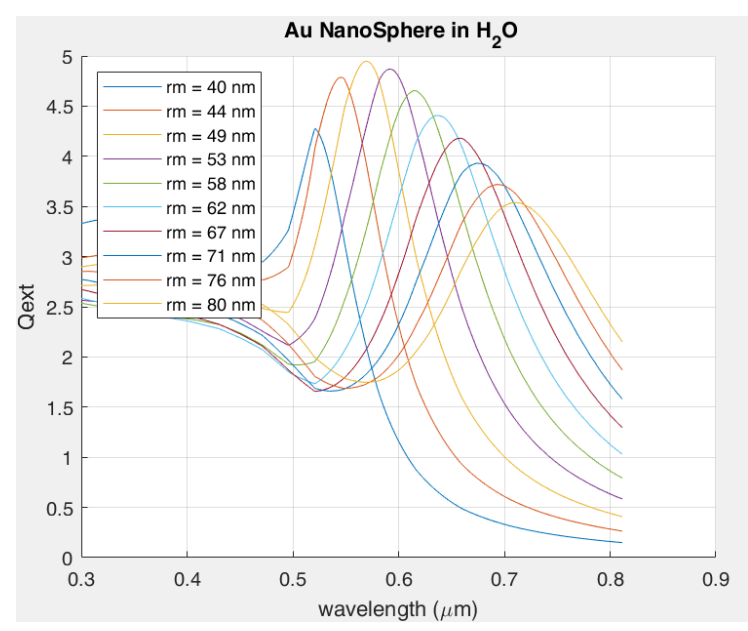

Figure 3. Extinction efficiency, calculated by Mie analysis, for gold nanospheres with $40 \mathrm{~nm}$ radius covered by a rGO cladding layer with thickness between 0 and $40 \mathrm{~nm}$ diluted in water.

\section{EXPERIMENTAL}

\section{A Preparation of $r G O$}

A modified Hummer's method [11] was used to synthesize $\mathrm{GO}$, in which graphite powder $(1.0 \mathrm{~g})$ is poured into a solution of $\mathrm{NaNO}_{3}(0.5 \mathrm{~g})$ in concentrated $\mathrm{H}_{2} \mathrm{SO}_{4}(30 \mathrm{~mL})$ and cooled to $0{ }^{\circ} \mathrm{C}$. $\mathrm{KMnO}_{4}(3.0 \mathrm{~g})$ is then added, during which the temperature of the mixture was maintained below $20^{\circ} \mathrm{C}$. Successively, the mixture is stirred at $35^{\circ} \mathrm{C}$ for $1 \mathrm{~h}$, and then diluted with deionized water $(46 \mathrm{~mL})$ by keeping the temperature at $85{ }^{\circ} \mathrm{C}$ and then increasing it to $100^{\circ} \mathrm{C}$ for 30 min. Warm deionized water $(140 \mathrm{~mL})$ is then added to the 
mixture, followed by the dropwise addition of a $50 \%$ aqueous solution of $\mathrm{H}_{2} \mathrm{O}_{2}(15 \mathrm{~mL})$, and the solution is stirred for 30 min. The mixture is then centrifuged and washed with a $5 \%$ aqueous solution of $\mathrm{HCl}(1 \mathrm{~L})$ to remove metal ions, followed by deionized water $(1 \mathrm{~L})$ to remove the acid. The dry GO powder is finally obtained after heating the filtrate for $12 \mathrm{~h}$ at $60^{\circ} \mathrm{C}$. Reduced Graphene Oxide (rGO) is synthesized by a solvothermal method. In a typical synthesis, GO is dispersed into ethylene glycol $(60 \mathrm{~mL})$ and sonicated for $1 \mathrm{~h}$, followed by the subsequent addition of aqueous $\mathrm{NH}_{3}(1.6 \mathrm{~mL})$, and stirred again for $1 \mathrm{~h}$. rGO is mixed with AuNPs by ball milling technique. Figure 4 displays the SEM (Scanning Electron Microscopy) image of the produced rGO.

\section{B. Preoparation of $r G O-A u N P$ s composite}

To prepare the rGO-AuNPs composite, a set of commercially available gold spherical nanoparticles with radius in the 20$50 \mathrm{~nm}$ range were used [12]. To achieve a uniform dispersion AuNPs and rGO were mixed through a mechanical process, with a ultrasonic bath at $44 \mathrm{kHz}$ for 15 minutes, and with a sonicator at $20 \mathrm{kHz}$, for 1 minute. The rGO concentration in the resulting colloidal solutions $(3 \mathrm{~mL})$ ranges from 0.5 to 4 $\mathrm{mg}$ for solutions containing $5.8 \times 10^{-5} \mathrm{~g} / \mathrm{mL}$ of gold.

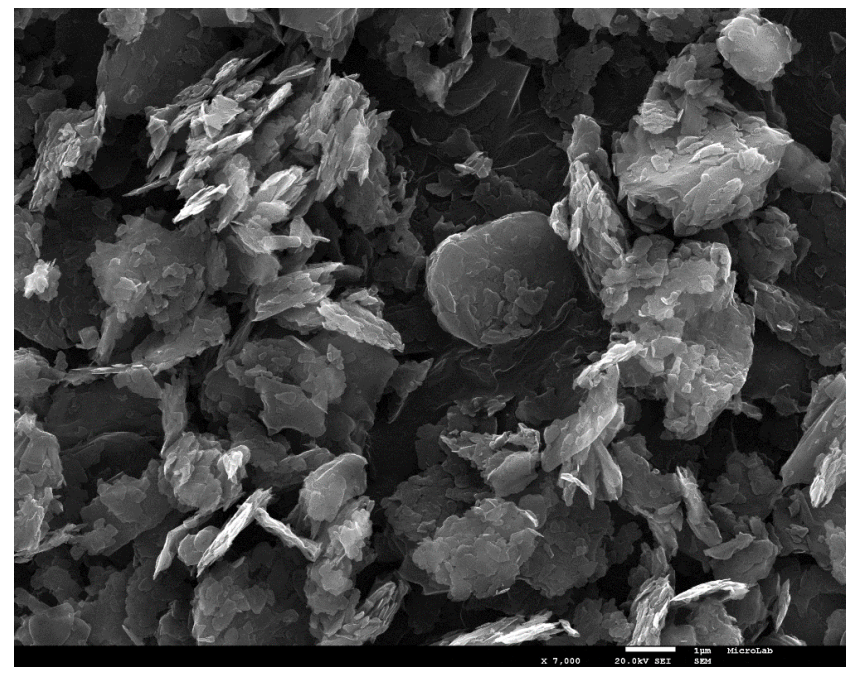

Figure 4. SEM image of the rGO used in the experimental measurements.

\section{UV-Vis measurements}

Although, no modification in the LSPR wavelength was detected for nanoparticles with radius less than $40 \mathrm{~nm}$, the influence of the rGO coupling to AuNPs could be clearly observed for $50 \mathrm{~nm}$ radius nanoparticles. Figure 5 shows the light absorption profile in the visible range, for different concentrations of rGO. The presence of rGO results in a general reduction of light transmission over the entire spectrum, in comparison to the solutions containing exclusively AuNPs. The LSPR peak, to be ascribed to the plasmonic behavior of the nanospheres is well defined in all the measured cases. The central wavelength for this plasmonic resonance is red shifted by the AuNP-rGO coupling, as reported in Figures 5 and 6, where the peak wavelength is plotted as a function of the rGO concentration. This is in accordance with the simulation results described in Figures 2 and 3 . Comparing the simulation and the experimental results we can conclude that even if there is no complete covering of the AuNP by the rGO (that is the cladding has by no means a uniform thickness, as simplistically assumed in the Mie analysis) the plasmonic resonance wavelength is affected by the difference in the refractive index introduced by the rGO. If no $\mathrm{rGO}$ is introduced, the observed LSPR is almost in perfect agreement with the simulated data. The red shifting caused by the increasing rGO concentration can be ascribed to a better and more complete cladding of the nanoparticles. It should be noted that the resonance peak remains unique and we cannot observe superposition of two separate peaks related to the cladded or not-cladded NPs.

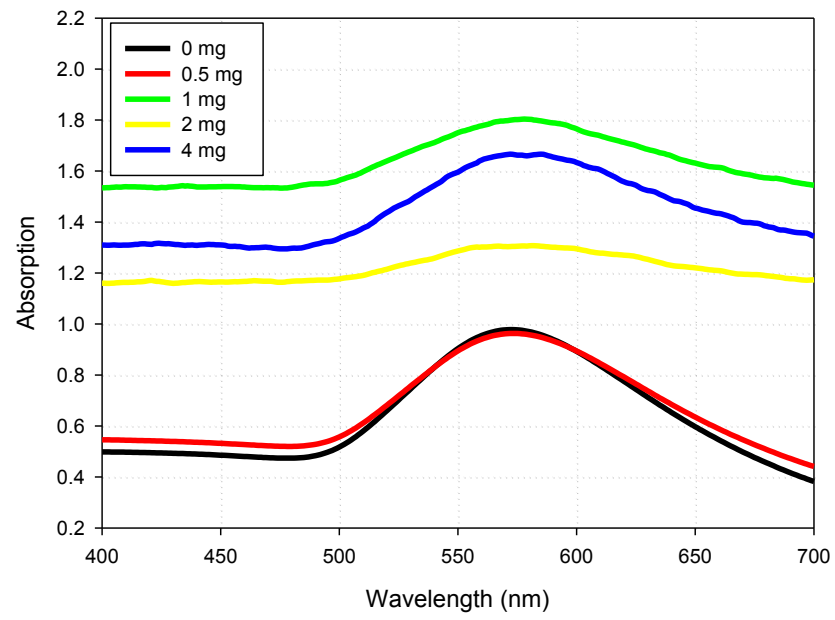

Figure 5. Measured absorption profile of the $\mathrm{rGO}+\mathrm{AuNP}$ colloidal solution with different concentration of rGO

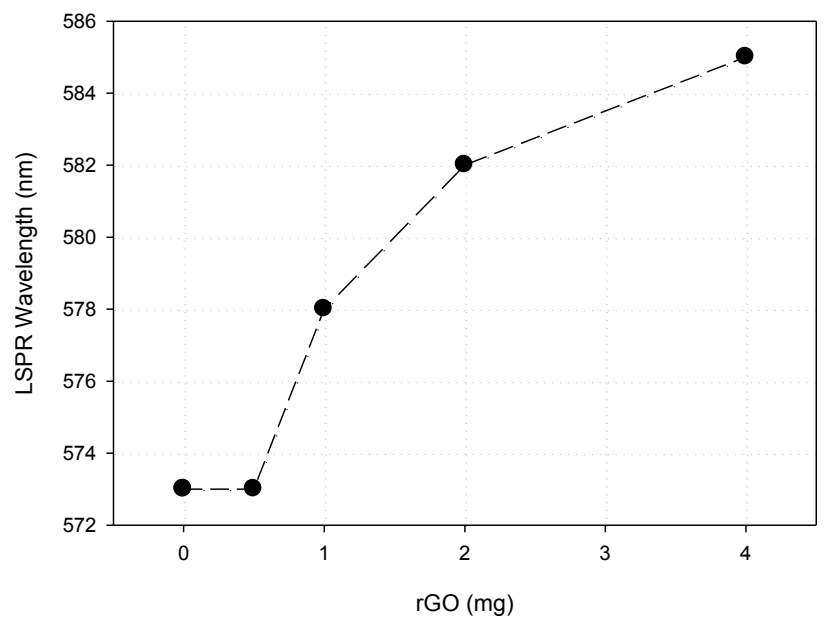

Figure 6. LSPR wavelength measured by UV-VIS absorption of $\mathrm{rGO}+\mathrm{AuNP}$ colloidal solution, as a function of the rGO concetration. Nanoparticle radius is $50 \mathrm{~nm}$. 


\section{CONCLUSION}

Considering that the AuNP-RGO composite is a promising candidate as a support for antibodies functionalization as the sensing layer if a photonic biosensor, we have demonstrated that it is possible to obtain a uniform mixture of AuNP and rGO using mechanical process like ultrasonic bath or ultrasonic sonication. Anyway, when the AuNP diameter is smaller than $100 \mathrm{~nm}$ no modification of the LSPR response can be observed. For $100 \mathrm{~nm}$ AuNP the mixture with rGO

[1] Pumera, M. (2011). "Graphene in biosensing", Materials today, 14(7-8), 308-315.

[2] Osváth, Z., Deák, A., Kertész, K., Molnár, G., Vértesy, G., Zámbó, D., ... \& Biró, L. P. (2015). "The structure and properties of graphene on gold nanoparticles". Nanoscale, 7(12), 5503-5509.

[3] Guo, W., Wu, L., Fan, K., Nie, D., He, W., Yang, J., ... \& Han, Z. (2017). "Reduced Graphene Oxide-Gold Nanoparticle Nanoframework as a Highly Selective Separation Material for Aflatoxins", Scientific Reports,7(1), 14484. [4] G.Mie, "Beiträge zur Optik trüber Medien, speziell kolloidaler Metallösungen", Annalen der physic, 330(3), 377-445 (1908).

[5] C. F.Bohren, D. R. Huffman, Absorption and scattering of light by small particles, John Wiley \& Sons (2008).

[6] E. D. Palik, Handbook of optical constants of solids (Vol. 3), Academic press (1998).

[7] R. L. Olmon, B. Slovick, T. W. Johnson, D. Shelton, S. H. Oh, G. D. Boreman, M. B Raschke "Optical dielectric function of gold". Physical Review B, 86(23), 235147 (2012). cause a red shift of the LSPR and the red shift depends on the RGO concentration. On the way for a biosensor application, future work will focus on an efficient method for functionalization with antibodies and the deposition of the composite on top of a photodiode photo-active surface.

\section{REFERENCES}

[8] K. M. McPeak, S. V. Jayanti, S. J. P. Kress, S. Meyer, S. Iotti, A. Rossinelli, and D. J. Norris. "Plasmonic films can easily be better: Rules and recipes", ACS Photonics 2, 326-333 (2015)

[9] Schmiedova, Veronika, et al. "Physical Properties Investigation of Reduced Graphene Oxide Thin Films Prepared by Material Inkjet Printing." Journal of Nanomaterials 2017 (2017).

[10] A. Fantoni, M. Fernandes, Y. Vygranenko, P. Louro, M. Vieira, E.C. Alegria, et alt... "A Simulation Study of Surface Plasmons in Metallic Nanoparticles: Dependence on the Properties of an Embedding a-Si: H Matrix”, Physica Status Solidi (a), 215(3), 1700487 (2018).

[11] Chen, J., Yao, B., Li, C., \& Shi, G. (2013). "An improved Hummers method for eco-friendly synthesis of graphene oxide". Carbon, 64, 225-229. [12] BBI Solutions, 73 Ty Glas Avenue Cardiff, UK https://www.bbisolutions.com 